\title{
Speeding up K-Means Algorithm by GPUs
}

\author{
You Li, Kaiyong Zhao, Xiaowen Chu, and Jiming Liu \\ Department of Computer Science \\ Hong Kong Baptist University \\ \{youli, kyzhao, chxw, jiming\}@comp.hkbu.edu.hk
}

\begin{abstract}
Cluster analysis plays a critical role in a wide variety of applications, but it is now facing the computational challenge due to the continuously increasing data volume. Parallel computing is one of the most promising solutions to overcoming the computational challenge. In this paper, we target at parallelizing $k$-Means, which is one of the most popular clustering algorithms, by using the widely available Graphics Processing Units (GPUs). Different from existing GPU-based $k$-Means algorithms, we observe that data dimension is an important factor that should be taken into consideration when parallelizing $k$-Means on GPUs. In particular, we use two different strategies for low-dimensional data sets and high-dimensional data sets respectively, in order to make the best use of the power of GPUs. For lowdimensional data sets, we exploit GPU on-chip registers to significantly decrease data access latency. For highdimensional data sets, we design a novel algorithm which simulates matrix multiplication and exploits GPU on-chip registers and also on-chip shared memory to achieve high compute-to-memory-access ratio. As a result, our GPU-based $k$-Means algorithm is three to eight times faster than the best reported GPU-based algorithm.
\end{abstract}

\section{Keywords-cluster, k-means, GPGPU, CUDA}

\section{INTRODUCTION}

Clustering is a method of unsupervised learning that partitions a set of data objects into clusters, such that intracluster similarity is maximized while inter-cluster similarity is minimized $[1,2]$. The $k$-Means algorithm is one of the most popular clustering algorithms and is widely used in a wide variety of fields such as statistical data analysis, pattern recognition, image analysis and bioinformatics $[3,4]$. It has been elected as one of top 10 data mining algorithms [5]. The running time of $k$-Means algorithm grows with the increase of the size and also the dimensionality of the data set. Hence clustering large-scale data sets is usually a timeconsuming task. Parallelizing $k$-Means is a promising approach to overcoming the challenge of the huge computational requirement [6-8]. In [6], P-CLUSTER has been designed for a cluster of computers with a client-server model in which a server process partitions data into blocks and sends the initial centroid list and blocks to each client. It has been further enhanced by pruning as much computation as possible while preserving the clustering quality [7]. In [8], the $k$-Means clustering algorithm has been parallelized by exploiting the inherent data-parallelism and utilizing message passing.

Recently, as a general-purpose and high performance parallel hardware, Graphics Processing Units (GPUs) develop continuously and provide another promising platform for parallelizing $k$-Means. GPUs are dedicated hardware for manipulating computer graphics. Due to the huge computing demand for real-time and high-definition 3D graphics, the GPUs have evolved into highly parallel many-core processors. The advances of computing power and memory bandwidth in GPUs have driven the development of general-purpose computing on GPUs (GPGPU).

In this paper, we design a parallel $k$-Means algorithm for GPUs by using a general-purpose parallel programming model, namely Compute Unified Device Architecture (CUDA) [9, 10]. CUDA has been used for speeding up a large number of applications [11, 12, 13, 14]. Some clustering algorithms have also been implemented on the GPUs, including $k$-Means. There are mainly three existing GPU-based $k$-Means algorithms: GPUMiner [15], $U V k$ Means [16], and HP_k-Means [17]. UV_k-Means achieves a speedup of ten to forty as compared with a four-threaded Minebench [18] running on a dual-core, hyper-threaded CPU. HP $k$-Means claims another speedup of two to four as compared with $U V \_k$-Means and twenty to seventy speedup as compared with GPUMiner [17]. These existing works have shown the promising high performance advantage of GPUs. However, the above GPU-based algorithms have not yet fully exploited the computing power of GPUs .

In this paper we conduct systematic research on parallelizing the $k$-Means using CUDA. Our first contribution is the observation that the dimensionality of the data set is an important factor to be considered. Our second contribution is the design, implementation, and evaluation of two different strategies. For low-dimensional data sets, we mainly utilize the GPU on-chip registers to minimize the memory access latency. Due to the limited size of on-chip registers, this method is not applicable to data sets with high dimensionality. For high-dimensional data sets, we design a novel and highly efficient algorithm that treats the most time-consuming part of $k$-Means as matrix multiplication, and then makes use of GPU on-chip shared memory together with on-chip registers. Our experimental results show that our $k$-Means performs much better than existing algorithms, for both low-dimensional data sets and high- 
dimensional data sets.

The rest of this paper is organized as follows. Section II introduces the GPU architecture and existing GPU-based $k$ Means algorithms. Section III presents our design of parallel $k$-Means algorithm on GPUs. Section IV presents our experimental results, and Section V concludes the paper and presents some future work.

\section{RELATED WORK}

To the best of our knowledge, there are mainly three existing GPU-based $k$-Means algorithms, namely $U V$ Means, GPUMiner, and HP_k-Means. We first briefly introduce the GPU architecture, and then review these three existing GPU-based $k$-Means algorithms.

\section{A. The GPU architecture}

We take NVIDIA GTX280 as an example to show the GPU architecture. GTX 280 has 30 Streaming Multiprocessors (SMs), and each SM has 8 Scalar Processors (SPs), resulting a total of 240 processor cores. The SMs have a Single-Instruction Multiple-Thread (SIMT) architecture: At any given clock cycle, each SP executes the same instruction, but operates on different data. Each SM has four different types of on-chip memory, namely registers, shared memory, constant cache, and texture cache, as shown in Fig.1. Constant cache and texture cache are both read-only memories shared by all SPs, but with very limited size. Off-chip memories such as local memory and global memory have relatively long access latency, usually 400 to 600 clock cycles [10]. The properties of the different types of have been summarized in $[10,12]$. In general, the scarce shared memory should be carefully utilized to amortize the global memory latency cost.

In CUDA model, the GPU is regarded as a coprocessor capable of executing a great number of threads in parallel. A single source program includes host codes running on CPU and also kernel codes running on the GPU. Computeintensive and data-parallel kernel codes run on the GPU. The threads are organized into thread blocks, and each block of threads are executed concurrently on one SM. Threads in a thread block can share data through the shared memory and can perform barrier synchronization. But there is no synchronization mechanism for different thread blocks besides terminating the kernel. Another important concept in CUDA is warp, which is formed by 32 parallel threads and is the scheduling unit of each SM. When a warp stalls, the SM can schedule another warp to execute. A warp executes one instruction at a time, so full efficiency can only be achieved when all 32 threads in the warp have the same execution path. There are two consequences: first, if the threads in a warp have different execution paths due to conditional branch, the warp will serially execute each branch which increases the total time of instructions executed for this warp; second, if the number of threads in a block is not a multiple of warp size, the remaining instruction cycles will be wasted. Besides, when accessing the memory, half-warp executes as a group, which has 16 threads. If the half-warp threads access the coalesced data, the access operation will perform within one instruction cycle. Otherwise, the access operation will occupy up to 16 instruction cycles.

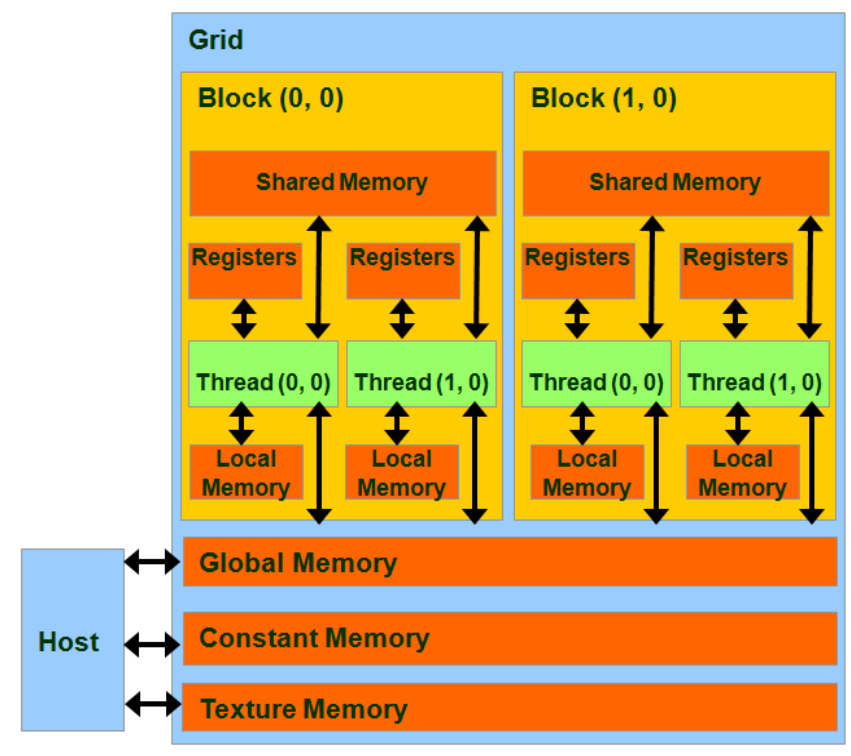

Figure 1. Hardware architecture of the GPU

\section{B. UV_k-Means}

In the $U V \_k$-Means, in order to avoid the long time latency of global memory access, it copies all the data to the texture memory, which uses a cache mechanism. Then, it uses constant memory to store $k$ centroids, which is also more efficient than using global memory. Each thread is responsible for one data point, finding the nearest centroid; each block has 256 threads, and the grid has $n / 256$ blocks.

The work flow of $U V \_k$-Means is straightforward. First, each thread calculates the distance from one corresponding data point to every centroid and finds the minimum distance and corresponding centroid. Second, each block calculates a temporary centroid set based on a subset of data points, and each thread calculates one dimension of the temp centroid. Third, the temporal centroid sets are copied from GPU to $\mathrm{CPU}$, and then the final new centroid set is calculated on CPU.

$U V \_$-Means has achieved a speedup of twenty to forty over the single-thread CPU-based $k$-Means in our experiment. This speedup is mainly achieved by assigning each data point to one thread and utilizing the cache mechanism to get a high reading efficiency. However, the efficiency could be further improved by other memory access mechanisms such as registers and shared memory.

\section{GPUMiner}

GPUMiner stores all input data in the global memory, and loads $k$ centroids to the shared memory. Each block has 128 threads, and the grid has $n / 128$ blocks. The main characteristic of GPUMiner is the design of a bitmap. The workflow of GPUMiner is as follows. First, each thread calculates the distance from one data point to every centroid, and changes the suitable bit into true in the bit 
array, which stores the nearest centroid for each data point. Second, each thread is responsible for one centroid, finds all the corresponding data points from the bitmap and takes the mean of those data points as the new centroids.

The main problem of GPUMiner is the poor utilization of memory in GPU, since GPUMiner accesses most of the data (input data point) from global memory. As pointed out in [17], bitmap approach is elegant in expressing the problem, but it is not a good method for high performance, since bitmap takes more space when $k$ is large and requires more shared memory.

\section{HP kMeans}

$H P \_$-Means is by far the most efficient $k$-Means algorithm on GPUs [17]. However, the details of $H P k$ Means are not reported in the paper. In general, $H^{-} k$ Means considers the GPU memory hierarchy: firstly, it arranges the data to make the best use of the memory bandwidth; secondly, it uses constant and texture memory to utilize the cache mechanism; thirdly, it uses shared memory for the data that will be read multiple times.

\section{DESIGN OF PARALLEL $K$-MEANS ON GPUS}

The $k$-Means algorithm is one of the most popular clustering methods. Given a set of $n$ data points $R=\left\{r_{l}\right.$, $\left.r_{2}, \ldots, r_{n}\right\}$ in a $d$ dimensional space, the task of $k$-Means is to partition $R$ into $k$ clusters $(k<n) S=\left\{S_{1}, S_{2}, \ldots, S_{k}\right\}$ such that $\sum_{i=1}^{k} \sum_{x_{j} \in S_{i}}\left\|x_{j}-\mu_{i}\right\|^{2}$ is minimized, where $\mu_{i}$ is the centroid of $S_{i}$.

The $k$-Means algorithm iteratively partitions a given dataset into $k$ clusters. It first selects $k$ data points as the initial centroids, which could be the first $k$ data points in the data set, or a set of randomly chosen $k$ data points. Then the algorithm iterates as follows: (1) Compute the Euclidean distance between each pair of data point and the centroid, and then assign each data point to its closest centroid; (2) Find the new centroids by taking the mean of all the data points in each cluster. The iteration terminates when the changes in the centroids are less than some threshold or the number of iterations reaches a predefined threshold. The whole process is shown in Algorithm 1.

The computational complexity of a single round of $k$ Means is $\mathrm{O}(n k d+n k+n d)$ : lines $2-6$ has a complexity of $\mathrm{O}(n k d)$; lines 7-14 has a complexity of $\mathrm{O}(n k)$; and line $15-$ 17 has a complexity of $\mathrm{O}(n d)$. According to the value of data dimension $d$, we design two different GPU-based algorithms, one for low-dimensional data sets and another one for high-dimensional data sets. For low-dimensional data sets, we mainly exploit on-chip registers to minimize the latency of data access. For high-dimensional data sets, we adopt the shared memory and apply the most efficient reduction method. Step (2) has a relatively low computational complexity of $\mathrm{O}(n d)$, and it is difficult to be fully parallelized due to the write conflict. So we let GPU handle part of the task that is worthy to be performed on GPU, and then send the remaining part to $\mathrm{CPU}$ for execution.

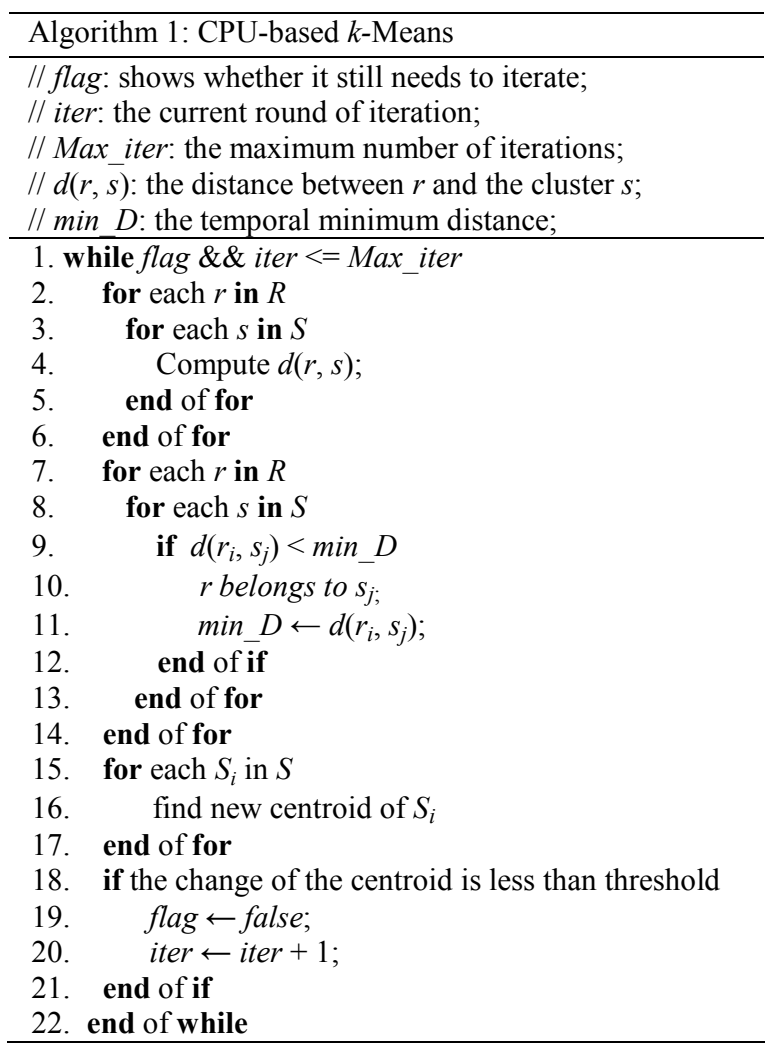

The following parts contain two subsections. Section A introduces algorithms for finding the closest centroid: Algorithm 2 is a CPU-based version; Algorithm 3 and 4 are two GPU-based versions for low- and high- dimensional data respectively. Section B illustrates the algorithms for computing new centroids: Algorithm 5 is based on CPU while Algorithm 6 uses both GPU and CPU.

\section{A. Finding closest centroid}

The CPU-based algorithm of finding closest centroid is shown in Algorithm 2. Since the algorithm computes the distance between each data point and each centroid, our first method to parallelize Algorithm 2 is dispatching one data point to one thread, and then each thread calculates the distance from one data point to all the centroids, and maintains the minimum distance and the corresponding centroid, as shown in Algorithm 3. Line 1 and 2 show how the algorithm designs the thread block and gird. Our experiments show that a block size of 256 results in better performance than block size of 32, 64 and 128. Line 3-4 tell how to calculate the position of the corresponding data point for each thread in global memory; line 5 loads the data point into the register; line 6-12 compute the distance and maintain the minimum one.

Algorithm 3 only has one loop instead of two loops in Algorithm 2, since the loop for $n$ data point has been dispatched to $n$ threads, which decreases the time consumption. It is worth pointing out that the key step of achieving high efficiency is loading the data points into the on-chip registers, which ensures that reading the data point 
from global memory happens only once when calculating the distances between the data point and $k$ centroids. Obviously, reading from register is much faster than reading from global memory. Besides, coalesced access to the global memory also decreases the reading latency. The experimental results in section IV show the advantage of Algorithm 3 compared with the best published results. However, the problem of Algorithm 3 is the limited size of the registers. In fact, users are not able to fully control the registers, and could only utilize registers when the data size is small enough. When the data points cannot be loaded into the registers as the data dimension grows, they will be stored in local memory, which will increase the reading latency and decrease the performance significantly.
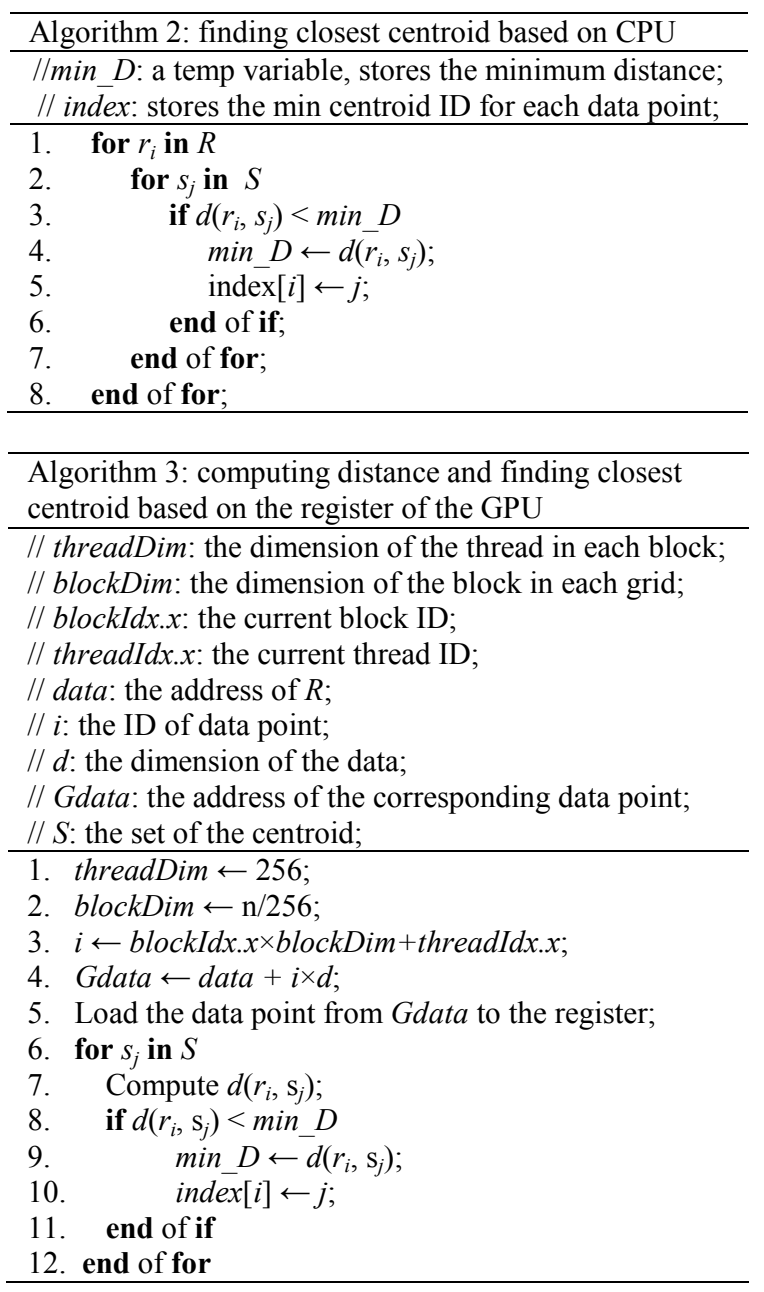

In fact, the input data point and the centroid could be viewed as two matrixes data $[n][d]$ and centroid $[d][k]$; the result distance could be denoted as $d[n][k]$; and the distance computing process shares the same flow as matrix multiplication. Based on this character, we design Algorithm 4 for high-dimensional data sets, by adopting the idea of matrix multiplication and utilizing registers and the shared memory together.

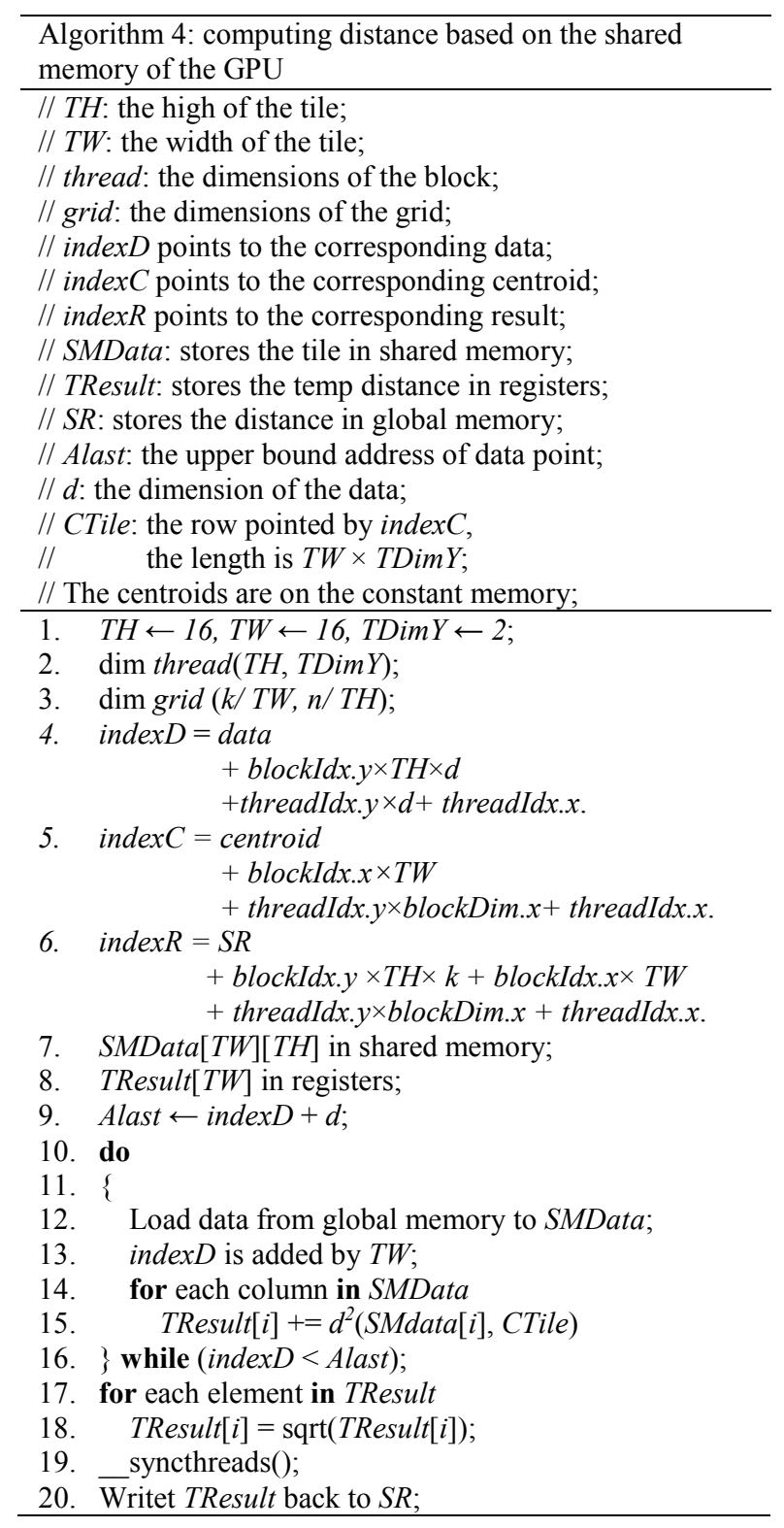

The main idea of Algorithm 4 is decreasing the global memory access times and latency by loading the data into the shared memory tile by tile. Thus, Algorithm 4 reads each data point from global memory only once, the same as Algorithm 3. The key point of Algorithm 4 is how to access the global memory and shared memory efficiently, which is achieved by adopting coalescing reading, accessing sixteen continuous address for the thread in a half warp to avoid the bank conflict. The details are described as follows.

Each block is in charge of computing a sub result matrix $S R[T W \times T \operatorname{Dim} Y][T H]$. Each block has $T H \times T \operatorname{Dim} Y$ number of threads, and each thread computes a column of $S R$. For each thread, indexD points to the right position of the data, which contains the following three parts as shown in line 4: data is the beginning address of the data set; since the height of the data is divided by $T H$, blockIdx. $y \times T H \times d$ is the address of the corresponding block; threadIdx. $y \times d$ adding 
threadIdx.x is the offset address inside the block.

In line 5 , indexC points to the right position in centroid, which also has three parts: centroid is the beginning address of the current centroid; blockIdx. $x \times T W$ points to the corresponding block address, since the width of the centroid is divided by the TW; threadIdx.y $\times$ blockDim.x adding threadIdx. $x$ points to the address of the current thread inside the block. Obviously, the threads in one block would access centroid in continues addresses, which is also called coalesced accessing and avoids bank conflict. index $R$ is calculated in the same way in line 6: the beginning address of the result, the row address, and the offset address inside the block for the current thread.

In the loop from line 11 to 16 , the algorithm loads a tile data from global memory to the shared memory, and computes the temp distance saved in TResult which are stored in on-chip registers; the loop ends when the whole row has been calculated, as shown in Fig.2.

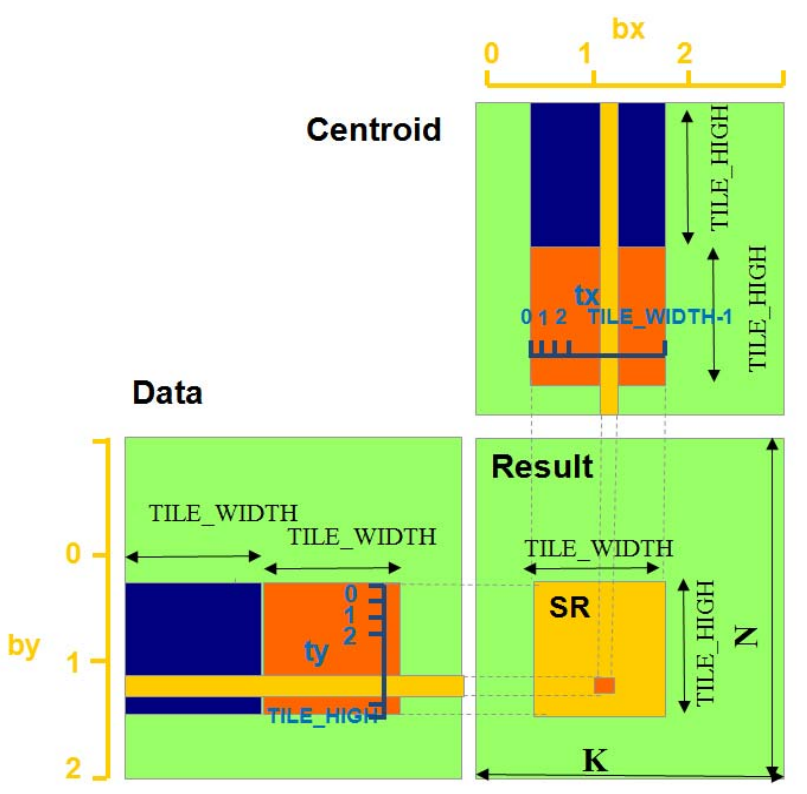

Figure 2. Tile-based distance computing process

After calculating the temp minimum distances from each sub matrix, we need to get the final minimum one. The computational complexity of CPU-based algorithm is $\mathrm{O}(n k)$. On GPU, if each data point is assigned to one thread, the computational complexity decreases to $\mathrm{O}(k)$. If each data point has $k$ threads, the complexity will be $\mathrm{O}(\log k)$. However, the cost of the parallel algorithm is $(k / \log k)$ and not efficient, which is measured by threads multiplying with complexity. In fact, according to Brent's theory, we can assign $\mathrm{O}(k / \log k)$ threads for each data point, finding the minimum distance from a $k$ array; each thread does $\mathrm{O}(\log k)$ sequential work; then all $\mathrm{O}(k / \log k)$ threads cooperate for $\mathrm{O}(\log k)$ steps. And the computational complexity is $\mathrm{O}(\log k)$ while the cost is $\mathrm{O}(k)$. This paper will not discuss the implementation in detail but adopt Brent's theory.

\section{B. Computing new centroids}

The result of finding the closest centroid is an array index $[n]$, which stores the closest centroid for each data point. The data points belonging to the same centroid constitute one cluster. Calculating the new centroids is by taking the mean of all the data points in each cluster. As shown in Algorithm 5, the computational complexity is $\mathrm{O}(n d+k d)$, and it is difficult to be fully parallelized. Since if we assign each data point to a thread, it will generate write conflict when adding the data to the shared centroid. On the other hand, if we assign each centroid to a thread, the computing power of the GPU cannot be fully utilized.
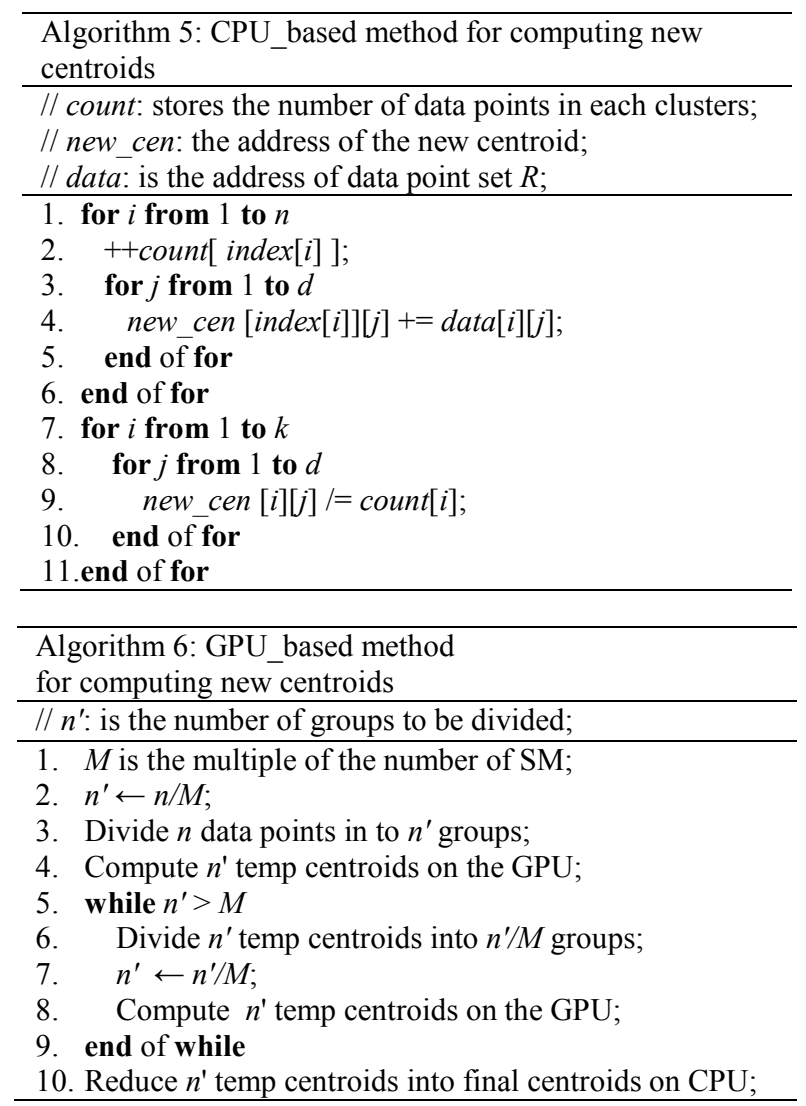

In this paper, we design an algorithm, which adopts the "divide and conquer" strategy: divide the data into groups; reduce each group and get temp centroids; then divide the temp centroids and reduce iteratively on the GPU until $n^{\prime}$ is smaller than $M$, which means the GPU has no advantage than the CPU for further computing; calculate the final centroids on the CPU, as shown in Algorithm 6. By dividing the data into groups, the write conflict decreases, since each group writes its own temp centroids and has no influence to other groups. And it is still a profitable method when we consider the additional data transfer through our experiments (see section IV). Besides, it is necessary to point out that $M$ in Algorithm 6 line 1 should be a multiple of the number of SM, which can ensure high schedule efficiency on the GPU. 


\section{EXPERIMENTS}

We have implemented our parallel $k$-Means algorithm using CUDA version 2.3. Our experiments were conducted on a PC with an NVIDIA GTX280 GPU and an Intel(R) Core(TM) i5 CPU. GTX 280 has 30 SIMD multiprocessors, and each one contains eight processors and performs at $1.29 \mathrm{GHz}$. The memory of the GPU is $1 \mathrm{~GB}$ with the peak bandwidth of $141.7 \mathrm{~GB} / \mathrm{sec}$. The CPU has four cores running at $2.67 \mathrm{GHz}$. The main memory is $8 \mathrm{~GB}$ with the peak bandwidth of $5.6 \mathrm{~GB} / \mathrm{sec}$. We calculate the time of the application after the file $\mathrm{I} / \mathrm{O}$, in order to show the speedup effect more clearly.

The experiments contain two parts: first, we compare our results with the best published results of HP_k-Means, which is mainly on low-dimensional data sets. Second, we compare our $k$-Means with $U V \_k$-Means and GMiner on high-dimensional data sets. Each of the experiments is repeated ten times and the average results are reported.

\section{A. On low-dimensional data sets}

Here we choose exactly the same data sets with $H P \_k$ Means as follows: $n$ has two values, two million and four million; $k$ has two values, one hundred and four hundred; $d$ also has two values, two and eight. Each dimension is a single-precision floating point number, and generated randomly. The iteration time is fifty, and the algorithm will stop after fifty rounds, no matter what the change of the centroid is. Besides, the speeds of HP_k-Means, $U V_{-} k$ Means and GPUMiner are extracted from [17]. As we use the same experimental configurations as [17], our comparisons are fair and reasonable.

As shown in Table 1, our $k$-Means is the most efficient one among the four algorithms. It is three to eight times faster than the best published results from $H P \_k$-Means, ten to twenty faster than $U V k$-Means and one hundred to three hundred faster than GPUMiner. Since HP_k-Means only provides some optimization rules without publishing the source code, we mainly discuss the difference between our $k$-Means and $U V \_k$-Means.

The workflows of the two algorithms are very similar: each thread finds the minimum centroid for each data point. The main difference is the memory utilization: $U V \_$-Means puts the data on the texture and puts the centroids on the constant; our $k$-Means firstly loads the data on the register, and reads the data from the register each time when calculating the distance from each centroid, resulting in a low global memory access times and latency, since reading from register is by far faster than reading from other memories.

We also analyze the GFLOPS of each algorithm. Since the computational complexity of finding the closest centroid is $\mathrm{O}(n k d+n k)$, which is much larger than that of computing the new centroid ( $\mathrm{O}(n d))$, a reasonable approximation on the total number of operations can be obtained by:

$$
O P=n \times k \times(d+d+d-1) \times \text { iter }
$$

For each data point, the first $d$ in Eq. (1) is the number of subtractions; the second $d$ is the number of multiplications; the third term $d-1$ is the number of additions; and iter is the number of iterations. Take the $7^{\text {th }}$ row of Table 1 as an example (i.e., $n=4$ million, $k=100, d=8$ ), the number of operations is $4.6 \times 10^{11}$. So the GFLOPS of each algorithm are: 676, 93, 36 and 1.2. The maximum GFLOPS of GTX 280 is around 933, and our algorithm has achieved $72 \%$ of the peak GPU computing power, which could also show the advantage of our algorithm. We visually show the time and GLOPS in Fig.3, for the setting of the $7^{\text {th }}$ line in Table 1

Table 1: Speed of $k$-Means on low-dimensional data, in second

\begin{tabular}{cccccrr}
\hline$n$ & $k$ & $d$ & $\begin{array}{c}\text { Our } \\
k \text {-Means }\end{array}$ & $\begin{array}{c}H P \\
k \text {-Means }\end{array}$ & $\begin{array}{c}U \text {-Means } \\
\text { kiner }\end{array}$ & $\begin{array}{c}\text { MPU } \\
\text { Miner }\end{array}$ \\
\hline 2 & 100 & 2 & 0.22 & 1.45 & 2.84 & 61.39 \\
million & 400 & 2 & 0.79 & 2.16 & 5.96 & 63.46 \\
& 100 & 8 & 0.35 & 2.48 & 6.07 & 192.05 \\
& 400 & 8 & 1.23 & 4.53 & 16.32 & 226.79 \\
\hline 4 & 100 & 2 & 0.34 & 2.88 & 5.64 & 130.36 \\
million & 400 & 2 & 1.22 & 4.38 & 11.94 & 126.38 \\
& 100 & 8 & 0.68 & 4.95 & 12.85 & 383.41 \\
& 400 & 8 & 2.26 & 9.03 & 34.54 & 474.83 \\
\hline
\end{tabular}

Note: the time is in second. The hardware environment of HP is as follows: NVIDIA GTX280 GPU; Intel Xeon CPU, 2.33GHz; 4GB memory.

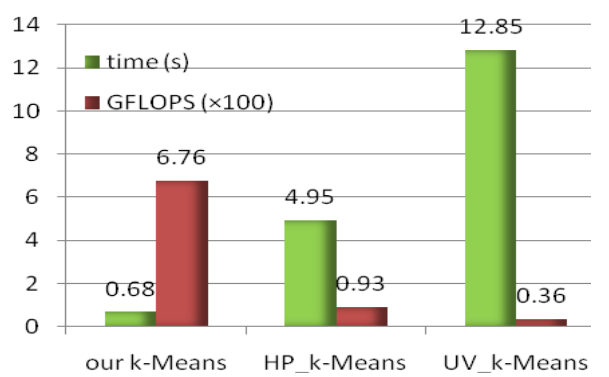

Figure 3. time and GFLOPS comparison on low-dimensional data

Table 2: Speed changes on dimension, in millisecond

\begin{tabular}{ccc}
\hline$d$ & $\begin{array}{l}\text { Finding the } \\
\text { closest centroid }\end{array}$ & $\begin{array}{c}\text { Computing } \\
\text { new centroids }\end{array}$ \\
\hline 2 & 72 & 64 \\
4 & 75 & 63 \\
6 & 83 & 64 \\
8 & 138 & 67 \\
10 & 405 & 64 \\
\hline Note: $n=10^{6}$, and $k=100$. &
\end{tabular}

Table 3: Speed changes on centroid, in millisecond

\begin{tabular}{ccc}
\hline$k$ & $\begin{array}{l}\text { Finding the } \\
\text { closest centroid }\end{array}$ & $\begin{array}{l}\text { Computing } \\
\text { new centroids }\end{array}$ \\
\hline 100 & 75 & 64 \\
200 & 138 & 66 \\
400 & 272 & 67 \\
\hline Note: $n=10^{6}$ and $d=4$ & &
\end{tabular}

Note: $n=10^{6}$, and $d=4$. 
As shown in Table 2, we can also observe that our $k$ Means is insensitive with dimension when the dimension is small, since the time differs a little when the dimension changes from 2 to 6 . As the dimension keeps growing, the time consumption grows with a higher speed, which is the main reason we design a new strategy for high-dimensional data. As shown in Table 3, when $k$ grows, the algorithm has to access the global memory more, which is proportional to $k$, and will spend more time. The computing time also grows with the increase of $n$, as shown in Table 4 . Besides, we could also see that the time consumption of "computing new centroids" changes slightly when the parameters change, due to the low computational complexity and our "divide and conquer" Algorithm 6.

Table 4: Speed changes on $n$, in millisecond

\begin{tabular}{ccc}
\hline$n$ & $\begin{array}{l}\text { Finding the } \\
\text { closest centroid }\end{array}$ & $\begin{array}{l}\text { Computing } \\
\text { new centroids }\end{array}$ \\
\hline $1,024,000$ & 77 & 60 \\
$2,048,000$ & 130 & 66 \\
$3,072,000$ & 512 & 62 \\
$4,096,000$ & 284 & 64 \\
$6,144,000$ & 431 & 69 \\
$8,192,000$ & 567 & 68 \\
\hline Note: $d=4$, and $k=100$. &
\end{tabular}

In fact, through our experiment, when the dimension is larger than sixteen, the data point cannot be loaded into the register, and the speed decreases sharply because of accessing the local memory. So, we use Algorithm 5, shared memory based algorithm to deal with the high-dimensional data.

\section{B. On high-dimensional data sets}

Here we use the data from the KDD Cup 1999 [19], and choose two data sets, with 51200 and 494080 data points respectively. Each data point contains 34 features, and each one is floating point. The default values of $k$ and iteration time are 32 and 100, respectively. Since HP_k-Means does not report any experimental results on high-dimensional data sets, we can only compare our algorithm with GPUMiner and UV_k-Means.

The comparison results are shown in Table 5. Our $k$ Means is four to eight times faster than $U V \_k$-Means, ten to forty times faster than GPUMiner, and one hundred to two hundred times faster than the CPU based $k$-Means algorithm developed by us.

When dealing with high-dimensional data, i.e., $d$ is larger than sixteen, our algorithm loads the data tile by tile into the shared memory. Thus it accesses the global memory only once for each data point. $U V \_k$-Means adopts texture to store the data point and decreases the global memory reading latency. However, it depends on the cache mechanism, and if the cache missing grows, the efficiency would decrease. On the other hand, the shared memory could perform more stably.
Table 5: Speed of $k$-Means on high-dimensional data, in second

\begin{tabular}{rlllc}
\hline Data set & $\begin{array}{l}\text { Our } \\
k \text {-Means }\end{array}$ & $\begin{array}{l}\text { UV } \\
k \text {-Means }\end{array}$ & $\begin{array}{l}\text { GPU } \\
\text { Miner }\end{array}$ & $\begin{array}{l}\text { CPU } \\
k \text {-Means }\end{array}$ \\
\hline 51,200 & 0.34 & 1.86 & 4.26 & 35.79 \\
494,080 & 1.15 & 8.67 & 40.6 & 224.47 \\
\hline
\end{tabular}

Table 6: Time distribution of our $k$-Means algorithm, in second

\begin{tabular}{cccc}
\hline Function/data set & $\begin{array}{l}\text { Finding the } \\
\text { closest centroid }\end{array}$ & $\begin{array}{l}\text { Computing } \\
\text { new centroids }\end{array}$ \\
\hline \multirow{2}{5}{51,200} & GPU & 0.10 & 0.25 \\
& CPU & 33.5 & 2.28 \\
\hline \multirow{2}{*}{94,080} & GPU & 0.87 & 0.24 \\
& CPU & 207.78 & 16.67 \\
\hline
\end{tabular}

Table 7: Speed changes on $k$, in millisecond

\begin{tabular}{ccc}
\hline$k$ & $\begin{array}{l}\text { Finding the } \\
\text { closest centroid }\end{array}$ & $\begin{array}{l}\text { Computing } \\
\text { new centroids }\end{array}$ \\
\hline 32 & 99 & 248 \\
64 & 138 & 251 \\
128 & 167 & 250 \\
256 & 204 & 252 \\
512 & 637 & 253 \\
\hline Note: $n=51,200$, and $d=34$.
\end{tabular}

Table 8: Speed changes on $n$, in millisecond

\begin{tabular}{rcc}
\hline \multicolumn{1}{c}{$n$} & $\begin{array}{c}\text { Finding the } \\
\text { closest centroid }\end{array}$ & $\begin{array}{c}\text { Computing } \\
\text { new centroids }\end{array}$ \\
\hline 51,200 & 99 & 248 \\
102,400 & 184 & 247 \\
204,800 & 340 & 249 \\
409,600 & 660 & 254 \\
819,200 & 1,318 & 259 \\
\hline
\end{tabular}

Note: $k=32$, and $d=34$.

Table 9: Speed changes on $d$, in millisecond

\begin{tabular}{ccc}
\hline$d$ & $\begin{array}{c}\text { Finding the } \\
\text { closest centroid }\end{array}$ & $\begin{array}{c}\text { Computing } \\
\text { new centroids }\end{array}$ \\
\hline 32 & 97 & 231 \\
64 & 127 & 276 \\
96 & 158 & 289 \\
128 & 185 & 290 \\
160 & 220 & 303 \\
\hline Note: $k=32$, and $n=51,200$. &
\end{tabular}

As shown in Table 6, finding the closest centroid achieves a speedup of forty to two hundred compared with our CPU-based algorithm, while computing new centroid achieves a speedup around ten, which further prove the advantage of our algorithm. The GFLOPS of the second line in Table 5 are 137, 18, 4 for our $k$-Means, $U V \_k$-Means and GPUMiner, as shown in Fig.4. Compared with Fig.3, the GFLOPS of our $k$-Means decreases, since the time consumption on computing new centroids occupies relative more percentage, which does not have a good speed-up effect. Besides, we also further compare Algorithm 3 and 4: 
when $n$ and $k$ are one million and one hundred, Algorithm 3 needs 1.5 seconds to deal with a 16 dimensional data set, while Algorithm 4 needs 1.5 to deal with a 32 dimensional data, which proves that it is necessary to design two strategies for different dimensional data.

As shown in Table 7 and 8, the time consumption shares the same trend in Table 3 and 4, which is appropriately linear with $k$ and $n$. Table 9 shows the speed changes on dimension, and the time of "finding the closest centroid" is also linear with $d$.

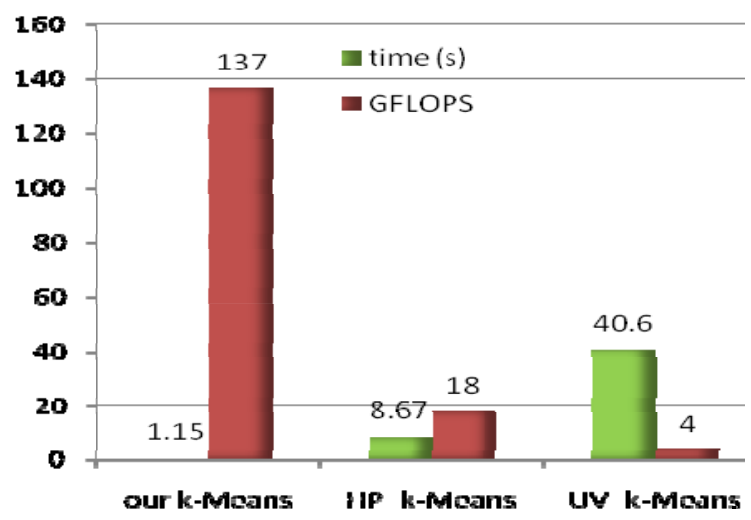

Figure 4. time and GFLOPS comparison on high-dimensional data

\section{CONCLUSIONS AND FUTURE WORK}

In this paper, we proposed a GPU-based $k$-Means algorithm. It presents mainly two novel ideas: first, based on the dimensionality of the data set, our $k$-Means algorithm chooses one from two different strategies. For lowdimensional data set, our algorithm utilizes GPU registers and achieves a speedup of three to eight over HP_k-Means. For high-dimensional data, our algorithm firstly observes the similarity between $k$-Means and matrix multiplication, then adopts shared memory and registers to avoid multiple accesses of the global memory, and finally achieves a speedup of four to eight as compared with $U V k$-Means.

The algorithm presented in this paper could deal with a finite scale of data set, the size of which is limited by the global memory size of the GPU. Consequently, when the data set is larger than it, new strategies have to be designed. A possible method is to divide the data set into several parts, each of which could be loaded into global memory of the GPU. If the data volume becomes even larger, a GPU cluster may be used which will introduce new optimization challenges.

\section{ACKNOWLEDGMENT}

This work is supported by FRG Grant: FRG2/08-09/098 from Hong Kong Baptist University.

\section{REFERENCES}

[1] P.-N. Tan, M. Steinbach, and V. Kumar, Introduction to Data Mining, Addison-Wesley Companion Book Site 2006.
[2] A. K. Jain and R. C. Dubes, Algorithms for Clustering Data, Prentice-Hall, 1988.

[3] X. Wang and M. Leeser, "K-means Clustering for Multispectral Images Using Floating-Point Divide," in Proceedings of the 15th Annual IEEE Symposium on FieldProgrammable Custom Computing Machines, 2007.

[4] H. Zhou and Y. Liu, "Accurate Integration of Multi-view Range Images Using $K$-means Clustering," Pattern Recogn., vol. 41, pp. 152-175, 2008.

[5] X. Wu, V. Kumar, J. R. Quinlan, J. Ghosh, Q. Yang, H. Motoda, G. J. McLachlan, A. Ng, B. Liu, P. S. Yu, Z.-H. Zhou, M. Steinbach, D. J. Hand, and D. Steinberg, "Top 10 Algorithms in Data Mining," Knowledge Information Systems, vol. 14, pp. 1-37, 2008.

[6] D. Judd, P. K. McKinley, and A. K. Jain, "Large-Scale Parallel Data Clustering," in Proceedings of the International Conference on Pattern Recognition (ICPR'96) Volume IV, 1996.

[7] D. Judd, P. K. McKinley, and A. K. Jain, "Large-Scale Parallel Data Clustering," IEEE Trans. Pattern Anal. Mach. Intell., vol. 20, pp. 871-876, 1998.

[8] I. S. Dhillon and D. S. Modha, "A Data-Clustering Algorithm on Distributed Memory Multiprocessors," in Revised Papers from Large-Scale Parallel Data Mining, Workshop on LargeScale Parallel KDD Systems, SIGKDD: Springer-Verlag, 2000.

[9] NVIDIA CUDA: http://developer.nvidia.com/object/cuda.html.

[10] NVIDIA CUDA Compute Unified Device Architecture: Programming Guide, Version 2.0, June 2008.

[11] S. A. Manavski, "CUDA compatible GPU as an efficient hardware accelerator for AES cryptography," In Proceedings of IEEE International Conference on Signal Processing and Communication, Nov. 2007.

[12] S. Ryoo, C. I. Rodrigues, S. S. Baghsorkhi, S. S. Stone, D. B. Kirk, and W. Hwu, "Optimization Principles and Application Performance Evaluation of a Multithreaded GPU Using CUDA," in Proceedings of the 13th ACM SIGPLAN Symposium on Principles and practice of parallel programming Salt Lake City, UT, USA: ACM, 2008.

[13] X.-W. Chu, K. Zhao, and M. Wang, "Practical Random Linear Network Coding on GPUs", in Proceedings of IFIP Networking'09, Archen, Germany, May 2009.

[14] X.-W. Chu, K. Zhao, and M. Wang, "Massively Parallel Network Coding on GPUs," IEEE IPCCC'08, Austin, Texas, USA, Dec 2008.

[15] W. Fang, K. K. Lau, M. Lu, X. Xiao, C. K. Lam, P. Y. Yang, B. He, Q. Luo, P. V. Sande, and K. Yang, "Parallel Data Mining on Graphics Processors," Technical Report HKUSTCS08, 2008.

[16] S. Che, M. Boyer, J. Meng, D. Tarjan, J. W. Sheaffer, and K. Skadron, "A Performance Study of General-Purpose Applications on Graphics Processors Using CUDA," Journal of Parallel and Distributed Computing, 2008.

[17] R. Wu, B. Zhang, and M. Hsu, "Clustering billions of data points using GPUs," in UCHPC-MAW'09: Proceedings of the combined workshops on UnConventional high performance computing workshop plus memory access workshop, Ischia, Italy, 2009, pp. 1-6.

[18] J. Pisharath, Y. Liu, W.-k. Liao, A. Choudhary, G. Memik, and J. Parhi, "NU-MineBench 2.0," CUCIS Technical Report CUCIS-2005-08-01, Center for Ultra-Scale Computing and Information Security, Northwestern University, 2005.

[19] KDD Cup 1998 Data: http://kdd.ics.uci.edu/databases/ kddcup99/kddcup99.html 